Article

\title{
How Data Will Transform Industrial Processes: Crowdsensing, Crowdsourcing and Big Data as Pillars of Industry 4.0
}

\author{
Virginia Pilloni \\ Department of Electrical and Electronic Engineering (DIEE), University of Cagliari, 09123 Cagliari, Italy; \\ virginia.pilloni@diee.unica.it; Tel.: +39-070-675-5877
}

Received: 10 December 2017; Accepted: 24 February 2018; Published: 1 March 2018

\begin{abstract}
We are living in the era of the fourth industrial revolution, namely Industry 4.0. This paper presents the main aspects related to Industry 4.0, the technologies that will enable this revolution, and the main application domains that will be affected by it. The effects that the introduction of Internet of Things (IoT), Cyber-Physical Systems (CPS), crowdsensing, crowdsourcing, cloud computing and big data will have on industrial processes will be discussed. The main objectives will be represented by improvements in: production efficiency, quality and cost-effectiveness; workplace health and safety, as well as quality of working conditions; products' quality and availability, according to mass customisation requirements. The paper will further discuss the common denominator of these enhancements, i.e., data collection and analysis. As data and information will be crucial for Industry 4.0, crowdsensing and crowdsourcing will introduce new advantages and challenges, which will make most of the industrial processes easier with respect to traditional technologies.
\end{abstract}

Keywords: Industry 4.0; Internet of Things; crowdsourcing; crowdsensing

\section{Introduction}

In the last decade, Internet of Things (IoT) and Cyber Physical Systems (CPS) have become pervasive and made an impact on many aspects of everyday life [1,2]: concepts such as Smart Home, Smart Car and Smart City have become familiar also to non-technical users. The integration of embedded systems, ubiquitous Internet access and Machine-to-Machine (M2M) communications have paved the way for IoT and CPS to be introduced also in industrial environments [3], under the forms of Industrial Internet of Things (IIoT or I2oT) [4,5] and Cyber-Physical Production Systems (CPPS) [6]. As a consequence, the fourth industrial revolution, namely Industry 4.0 [7], is rapidly reshaping traditional industrial processes.

The first three industrial revolutions were characterised by technical innovations that aimed to improve production efficiency: the first industrial revolution was triggered by water and steam power; electricity and assembly lines enabled mass production in the second industrial revolution; in the third industrial revolution, the introduction of control automation and Programmable Logic Controllers (PLCs) gave a boost to factory production [8,9]. The fourth industrial revolution is mainly enabled by three innovation elements [3]: ubiquitous Internet access, M2M communications and advanced data analysis. The objective is not only improving production efficiency but also enabling the so-called mass customization [10], i.e., the mass production of personalised products by making use of modularised product design and flexible processes.

One of the keys to customer satisfaction is providing services and products with a high level of customisation. This is enabled by production processes that are increasingly corresponding to customers' expectations and needs, both in terms of timeliness and quality [11]. In order to fulfill these requirements, industry-related processes, from production to delivery, from warehousing to stock replenishment, need to be adequately designed so that resource usage is optimised, and faults and system downtimes 
are minimised. Furthermore, workplace health and safety, as well as the quality of working conditions, need to be ensured to improve work-life balance and, therefore, workers' happiness, according to the assumption that happier workers are more productive.

In this paper, all of these aspects will be analysed by considering how the use of new technologies related to IIoT and CPPS can enhance industrial processes. Since the main difference of this industrial revolution with respect to the previous ones is the focus on humans and how their perceptions affect industrial processes, this aspect will be thoroughly analysed. In Industry 4.0, workers and customers are an integral part and the core of most of the decision processes, both in terms of objective measurements of their activities and subjective feedback gathering. In this context, the use of crowdsensing and crowdsourcing introduces many advantages [12]. In crowdsensing and crowdsourcing, data are collected collaboratively by exploiting the pervasive presence of Internet-connected geolocated devices. This enables the collection of impressive amounts of data and the opportunity of analysing them to perform more advanced processes and applications.

The remainder of the paper is organised as follows. In Section 2, the background of the main enabling technologies that enabled the fourth industrial revolution are examined. Section 3 describes the applications that are expected to characterise Industry 4.0, subdivided into five application domains. Section 4 provides a discussion about the challenges that current technologies will have to face for being effectively integrated into industrial processes, despite their stringent requirements. The section ends with an overview of the opportunities that will be created. Section 5 focuses on the use of crowdsensing and crowdsourcing to enable the applications that characterise Industry 4.0. Finally, Section 6 draws final conclusions.

\section{Industry 4.0 Background}

The birth of the fourth industrial revolution, namely Industry 4.0, is assumed to coincide with the Industrie 4.0 initiative [13]. Industrie 4.0 is a strategic initiative included by the German government as part of its High-Tech Strategy 2020 Action Plan, according to which the Internet-based technologies are used to improve all industrial processes, from manufacturing to distribution, from sales to post-sales. In the same period, similar initiatives were undertaken by other countries, such as Internet + in China [11] and Industrial Internet in the USA [3]. All of them moved from the consideration that new paradigms such as Internet of Things (IoT), Cyber-Physical Systems (CPS), crowdsensing, crowdsourcing, cloud computing and big data, which were already being used to turn "normal" environments into smart environments (e.g., Smart Homes, Smart Cities), could be integrated with industry-related processes to enhance their performance.

Intelligence had been already introduced in industries thanks to embedded systems, i.e., systems made of devices where sensors and actuators are embedded and connected to a control unit via field buses [8]. However, traditional embedded systems are conceived as stand-alone systems. The technologies introduced by Industry 4.0 enable communication and cooperation among devices and stand-alone systems, so that a higher level of intelligence can be provided to industrial processes $[8,14]$. More specifically, there are two ways in which communication can provide intelligence to objects: (i) less capable objects can leverage the resources of smarter objects, whether they are located nearby or remotely (e.g., cloud computing). Accordingly, less powerful objects typical of the industrial scenario, such as sensors and RFID (Radio Frequency Identification) tags, can cooperate with other more powerful objects to perform smarter applications; (ii) communication can be used by objects that are already smart, such as smartphones, to "augment" their intelligence thanks to information collected by distributed objects, whether it is objective, coming from measures collected by sensors, or subjective, provided by humans. This is the concept on the basis of crowdsensing and crowdsourcing, where big amounts of data gathered by multiple nodes are processed to get more complex information.

According to these considerations, seamless ubiquitous Internet access, communication between intelligent machines and advanced analytics methods are to be considered the pillars of Industry 4.0 
revolution [3], fostered by the IIoT [4,5] and CPPS [6] paradigms. These components will be analysed in the following.

\subsection{Ubiquitous Internet Access}

Industrial environments have been characterised by devices connected through wired communication media working over industrial protocols [15]. Indeed, considering the stringent requirements related to the control of industrial processes, cables are the most straightforward options in terms of reliability, security, latency and resilience to interferences. Nevertheless, wireless networks offer significant advantages with respect to wired ones [16,17]:

- Since they are not limited by a cable, they are quick and easy to deploy, even for hard to reach and remote locations.

- They offer more flexibility and easily adapt to changes in the network configuration, thus improving scalability.

- They enable device mobility, providing real-time Internet access anywhere and anytime.

- They are available in personal smart devices such as smartphones, tablets, and smartwatches, which are equipped with a large number of sensors. Therefore, they can provide relevant information about the owner, enabling applications related to, for example, employee monitoring or customer feedback collection.

For these reasons, and in order to meet the requirements that are typical of industrial environments, specific Industrial Wireless Network (IWN) solutions have been developed. Compared to traditional wireless networks, IWNs have to address the following issues [17]:

- Interference and path loss: industrial environments are characterised by challenging conditions, due to the presence of dust, vibrations, heat, obstacles, critical temperatures and humidity levels. Furthermore, the presence of motors, metal obstacles and other wireless communications introduces severe signal interference.

- Latency: industrial processes are typically required to have real-time or quasi-real-time performance, hence latency has to be kept low. Nevertheless, higher energy amounts and costs are needed to reduce latency, and this might affect nodes' lifetime.

- Changing location: industrial processes may be location-dependent, meaning that node location may be a parameter for the process to be carried out. Therefore, node location should be always known, even when they are mobile. Accordingly, localization and tracing mechanisms need to be introduced.

- Security and privacy: since wireless signals can be easily intercepted, they are much more susceptible to security and privacy attacks (e.g., jamming, spoofing, packet sniffing) with respect to wired signals. For this reason, specific security and privacy-preservation technologies have been developed, so that industrial processes and employee/customer data are not threatened.

The evolution of wireless solutions to meet these requirements enables ubiquitous Internet access even for harsh scenarios and demanding processes such as the industrial ones. Therefore, everyone and everything will be connected: people to people, people to objects, people to services, and objects to objects [11]. A further evolution would be the introduction of hardware and software handover mechanisms, which would make seamless communication possible.

\subsection{Machine-to-Machine Communication}

The Industry 4.0 paradigm is based on machines, devices, logistics and humans connected to each other to exchange data, process them and make decisions based upon them, while appropriately coordinating in a ubiquitous and ad hoc manner thanks to M2M communications [15]. According to this vision, objects are equipped with communication capabilities that enable them to be autonomous and self-organizing [18]. As discussed in the previous subsection, Internet access is going to be 
ubiquitously available in industrial environments, thus providing connectivity to all the involved objects. Nevertheless, not only smart objects are going to be part of Industry 4.0. Thanks to the evolution of Micro-Electro-Mechanical Systems (MEMS) technology, tiny low-cost low-power sensors with communication interfaces have started to be embedded into the most various objects. Some examples are: RFID tags that are placed on products for supply chain management and energy harvesting sensors supplied by machinery vibration, used to monitor manufacturing processes. Since they are developed to energy-efficiently transmit data, they are not provided with high computational capabilities, thus relying on other objects' intelligence for their cooperation- and organization-related tasks. For this reason, M2M communication is ensured not only among physical objects but also among their digital counterparts residing in the cloud [19]. Data are then stored and/or processed in the cloud, which provides to the system the resources that it needs. In this case, since data cannot be immediately associated with the object that produced them, and their location and time, appropriate mechanisms have to be applied to ensure that integrity and consistency are maintained [17].

The choice of using local or remote resources basically depends on the application that has to be performed by the system and the location of the required resources, i.e., sensing and/or processing capabilities, and storage capacity [20]. If the required resources can be provided by objects that are located close to each other, objects can be able to form a connected group, preferably relying on short-range communication technologies. In this case, the management of resources should be carried out as close as possible to the point where they are used. On the other hand, if the objects that are involved in the application execution cannot directly contact local objects that can provide the required resources, other remote objects can be contacted. Depending on the position of these remote objects with respect to the core network, we can refer to fog/edge computing [21] or cloud computing technologies.

\subsection{Advanced Analytics}

As introduced in the previous subsections, ubiquitous Internet access, IoT, M2M communication, and cloud computing are among the enabling technologies of Industry 4.0. They are used to collect and handle huge amounts of data (big data) that can be used to achieve in-depth understanding, gain insights and perform discoveries for accurate decision-making that will guide industrial processes [7]. Wide ranges of data can be integrated for this purpose, both structured and unstructured, from historical to real-time, coming from the most diverse sources, e.g., sensors, machines, products, workers, and customers. In particular, people will be among the most valuable data sources in Industry 4.0, for example: feedback from customers will be used to improve mass customization [22] and data gathered from worker devices will be used for network collaboration [7]. In this context, crowdsensing and crowdsourcing, often referred to as mobile crowdsensing and mobile crowdsourcing, provide a new way to collect data collaboratively, by exploiting the pervasive presence of Internet-connected geolocated smart user devices, such as smartphones, smartwatches and tablets [12]. In crowdsensing [23], these devices serve as moving sensors to collect data from different places. In crowdsourcing [24], the objective is for users to complete a task, usually consisting of providing feedback. In the following, when no further differentiation is needed, MCS will be used to refer to both mobile crowdsensing and mobile crowdsourcing. Although these two novel paradigms are very powerful, as they enable the collection of massive amounts of useful local data and information in a cost-effective and scalable way, they have not been widely used in industries yet, due to the strict requirements that characterize this environment [12].

\section{Application Domains}

As emerged from the previous section, Industry 4.0 will address several issues that are typical of industrial environments. In fact, the main objective of Industry 4.0 is to improve the perceived quality of products according to customers' feedback, while optimizing the related industrial processes by increasing their flexibility, speed, productivity and quality [25]. Accordingly, the following application domains are the levers that will drive the development of Industry $4.0[9,12,26]$. Figure 1 shows 
the enabling technologies of Industry 4.0 and their related application domains. The subdivision of Industry 4.0 applications into application domains is depicted in Figure 2.

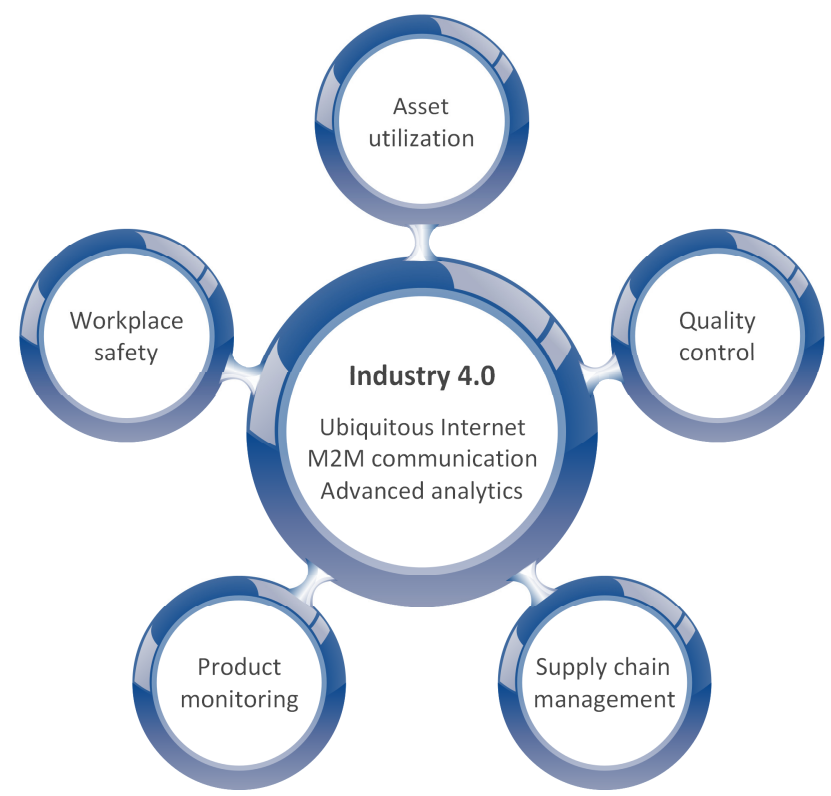

Figure 1. Enabling technologies and application domains in Industry 4.0.

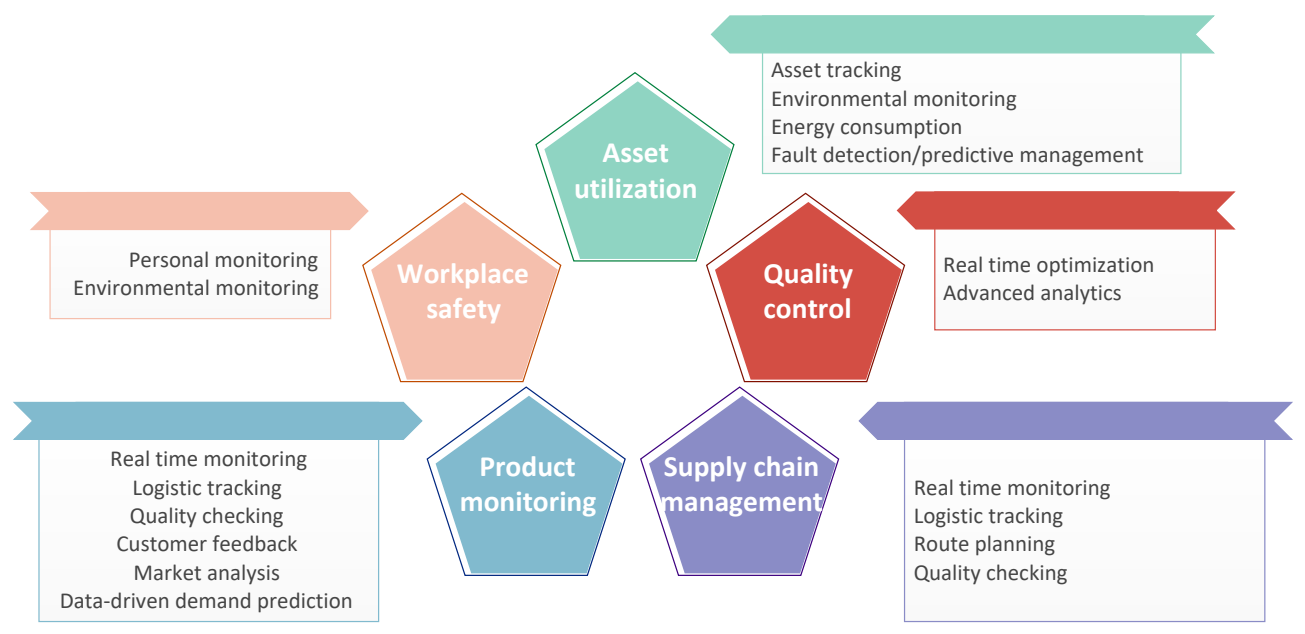

Figure 2. Application domains and related applications in Industry 4.0.

\subsection{Asset Utilization}

Thanks to ubiquitous Internet access and M2M communication, it will be possible to know anytime and anywhere the state of industrial assets. This is related not only to their current condition but also to their location. Some embedded sensors, such as RFID tags, will be used to locate assets that can be moved from one place to another, thus improving asset tracking and reducing inventory management costs and mistakes [27]. Furthermore, data gathered from sensors embedded into assets or workers' personal devices will be used for environmental monitoring, to ensure that working conditions are good [28]. Smart meters will be used to collect information on energy consumption, which can be later analysed to find optimal energy utilization patterns [10]. Analysis of data coming from asset monitoring will be used for fault detection and predictive maintenance purposes [29]. Based on 
the acquired information and also taking advantage of M2M communication, assets will be remotely controlled to improve industrial processes [30] .

\subsection{Quality Control in Manufacturing}

As introduced above, data collected during asset utilization monitoring can be analysed to improve the quality of industrial processes, e.g., through fault detection, predictive maintenance and energy consumption monitoring. Accordingly, critical conditions will be detected and real-time yield optimization solutions will be identified [31]. Statistical Process Control (SPC), Advanced Process Control (APC) and Six Sigma strategies can be used on such data to enhance process control [32]. Big data will play a key role, as advanced analysis techniques will be used to compare and interpolate data collected from sensors with related information that is made available on the cloud, so that more efficient solutions can be elaborated [33]. For example, data collected while monitoring some unusual behaviour of a machine can be analysed with data on the cloud to find similar patterns that suggest possible solutions already implemented by others in similar situations.

\subsection{Supply Chain Management}

Embedded sensor data will be used not only inside industries during manufacturing processes but also outside of them, in order to improve supply chain performance. Combining the RFID technology with ubiquitous Internet access, real-time monitoring of location and condition of products during their transit will be made possible [34]. Accordingly, logistic tracking will be made easier and less prone to mistakes [35]. Data about products, destinations, drivers and warehouse requirements will be analysed and processed along with data about traffic and weather conditions for optimal route planning [36]. Sensors will be also used to perform real-time quality checking [37], i.e., to monitor the condition of products, their packaging, and their location. This will be particularly useful to ensure that products with particular requirements, e.g., temperature for frozen food, are conveyed in the best possible conditions.

\subsection{Product Monitoring}

Directly connected to supply chain management is product monitoring, which refers to the activities that are associated with the product from the moment it actually becomes a product within the manufacturer's premises, to the one it is purchased and used by customers. This includes real-time monitoring of location and condition, logistic tracking and quality checking, which are explained above in the previous subsection. Besides the objective parameters related to the product quality, mainly regarding defect tracking, product monitoring also refers to the subjective assessment of their quality. To this end, customer feedback and market analysis are required $[38,39]$. Thanks to modern smart devices, these activities are performed in an easier, more efficient and cost-effective way: feedback will be gathered using the mobile crowdsourcing paradigm, data coming from customers will be integrated with market-related data and, based on them, advanced analysis techniques will be used to generate relevant KPIs (Key Performance Indicators). Furthermore, thanks to the integration of customer feedback with product tracking, data-driven demand prediction will be enabled [40]. Therefore, it will be possible to optimize production and supply chain processes.

\subsection{Workplace Safety}

Another important issue in the industrial environment is related to workers' safety. Indeed, workplace hazards and faults can be easily and promptly detected thanks to personal monitoring and environmental monitoring [41]. In Industry 4.0, enabling technologies for workplace safety are embedded sensors placed on assets and workers' personal devices that, thanks to M2M communications, can be used to raise an alarm as soon as a possible hazard is detected. For example, smart helmets [42,43] can be used and integrated with information coming from static sensors, in order to monitor $\mathrm{CO}_{2}, \mathrm{SO}_{2}, \mathrm{SH}_{4}$, wind speed, humidity and temperature. 


\section{Industry-Related Challenges and Opportunities}

In order for the application domains analysed in Section 3 to work properly, there are crucial requirements that characterise Industry 4.0 that need to be satisfied. As identified by the IEEE Time Sensitive Networks (TSN) Working Group (WG), the main challenges that need to be faced before the Industry 4.0 vision becomes a reality are $[7,44,45]$ :

- reduce latencies and ensure accuracy independently from the physical medium: the majority of industrial applications require real-time and deterministic responsiveness. This is already ensured by typical communication standards used in industrial scenarios (e.g., PROFINET, HART), but with the introduction of wireless communication standards and devices with heterogeneous characteristics, this performance might degrade. Indeed, existing communication standards that are not conceived for industrial applications need to be adapted in order to take these requirements into account, particularly introducing mechanisms for prioritization, time slot allocation and synchronization. Furthermore, the use of different communication standards needs to be performed seamlessly. These requirements need to be fulfilled even considering an increase in demand;

- perform fault tolerance without additional hardware: reliability of data flows needs to be ensured even in the event of faults and failures, without installing additional hardware that would nullify the benefit of using the existing hardware to perform the applications. To this aim, the IEEE TSN WG (Time-Sensitive Networking Working Group) is defining mechanisms that allow replication and redundant transmission of data over several disjunctive paths;

- support higher security, safety and privacy: with the introduction of heterogeneous communication media and protocols that are not conceived to work in an industrial environment, particularly wireless, it is necessary to study new mechanisms that ensure safety and security. It should be ensured that the production facilities and product itself do not threaten people and the environment. Product misuse and unauthorized access to production facilities need to be prevented. Furthermore, the acquisition of personal data about customers and workers introduces privacy issues that need to be taken into account when designing and implementing an Industry 4.0 application [46];

- provide interoperability of solutions from different manufacturers: existing industrial hardware mainly uses proprietary solutions to work and communicate with other devices. Indeed, one of the major known issues related to the use of heterogeneous devices is interoperability between newly installed devices and existing devices, particularly when existing devices are extremely efficient, reliable and expensive, and their replacement would be difficult. Therefore, a set of uniform standards needs to be developed so that a network between different factories and companies can be connected and integrated.

When all these challenges will be satisfactorily met, the following objectives are envisioned to represent the opportunities that will foster the spread of Industry 4.0 applications [7,13,47]:

- the improvement of industrial process management will lead to an improvement in the whole production, mainly given by the reduction in production time. The increased level of integration and data exchange will lead to an increase in the complexity of business processes and in the level of automation ensured. Flexibility will be improved, as processes will be made more agile. Dynamic and adaptive optimized decision-taking will improve productivity and efficiency: the lowest amount of resources will be used to produce the highest volume of products, while minimizing emissions due to production processes;

- improved working conditions will be ensured, both taking into account an improvement in security and safety, and in working time, work organisation, and work-life balance. Indeed, thanks to the improvements provided by the previous objective and to new technologies such as MCS and big data analysis, new innovative services will be provided. This will not only create new value opportunities but also enable diverse and flexible career paths that will allow people to work and remain productive 
for longer. Furthermore, this new flexibility will enable more flexible work organisation models, which will gradually meet the growing need of employees to strike a better balance between their work and private lives;

- new technologies, particularly with reference to MCS and advanced analysis, will be key not only to improve production and working conditions but also for an improvement in customer satisfaction. First off, optimized production processes and supply chain management will lead to a supply that perfectly fits the demand. Furthermore, individual, customer-specific criteria will be included in the design, configuration, ordering, planning, manufacturing and operation phases, also incorporating last-minute changes, indeed enabling mass customization to be implemented.

\section{Using MCS in Industry 4.0 Scenarios}

\subsection{Role of MCS in Industry 4.0}

One of the key concepts that recur when talking about Industry 4.0 is the management, transmission, analysis and storage of big amounts of data. Most of the complex processes described in the application domains of Section 3, as well as the three objectives of improving production, working conditions and customer satisfaction (see Section 4), are based on the processing and aggregation of data coming from several different sources. It is evident that data handling will be pivotal for the implementation of the industry-related complex processes.

From the previous Sections, three main data sources can be identified:

- assets and environmental monitoring. It is based on objective measurements performed by sensors, mainly static or with a limited mobility range;

- products and workers monitoring. It is based on objective measurements performed by sensors, mainly mobile and geolocated;

- workers and customers' feedback. It is based on the acquisition of subjective feedback, mainly mobile and geolocated.

Most of the applications described in Section 3 require a multitude of data to be enabled, data that are later processed according to big data principles, with specific engines such as those described in [48-50]. In order to collect these different types of unstructured data coming from such heterogeneous sources, the MCS paradigm can be used. As introduced in Section 2.3, MCS includes both mobile crowdsensing and mobile crowdsourcing. Mobile crowdsensing makes use of mobile sensors to collect data from a crowd of different sources. In mobile crowdsourcing, users are required to complete a task, usually consisting of providing feedback [12]. As shown in Figure 3, MCS introduces a number of advantages in industrial applications with respect to traditional sensor networks [12,51]: they make use of ubiquitous Internet access to provide mobile and scalable measures; they do not need additional dedicated devices to extend the existing industrial sensing system, as they use data coming from any device, especially personal devices; they enable the acquisition of subjective assessments, in addition to objective measurements; they can integrate human wisdom into machine intelligence; finally, industrial applications can benefit the information-sharing and decision-making processes of the whole industrial society.

Taking into account the type of data acquisition required for each industrial application analysed in Section 3, Table 1 compares MCS to traditional Industrial Sensor Networks (ISNs). MCS is based on mobile data acquisition, whether through objective acquisition made by sensors and subjective acquisition provided by users' personal devices. Therefore, while it is not always the best solution in asset utilization-related applications, it is particularly efficient, with respect to traditional ISNs, in the other applications, which are improved by mobile acquired data and subjective data. Furthermore, it enables the cost-effective acquisition of large amounts of data. This makes it not only the most efficient solution in quality control, supply chain management and workplace safety applications but also the only possible solution in some product monitoring applications, for which traditional solutions would be too complicated, expensive and slow to be implemented. 


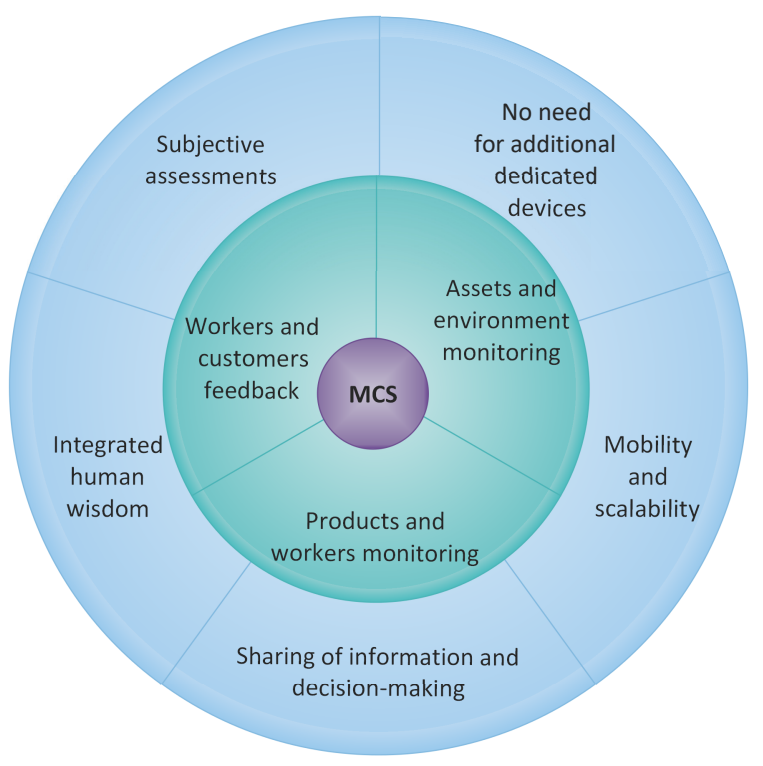

Figure 3. The advantages of using mobile crowdsensing and mobile crowdsourcing in Industry 4.0.

\subsection{MCS Architecture for Industry 4.0}

Based on the considerations of the previous sections, in order for MCS to be enabled in Industry 4.0 applications, there are some components that need to be included in traditional industrial communication systems [12,52-54]. Figure 4 shows an explanatory MCS-enabled Industry 4.0 architecture. The main components are explained in the following:

- MCS-enabled end device: it is a mobile device that is provided with communication interfaces and enough computation capabilities to run the services that enable MCS in them. In particular, specific interfaces to communicate with the components residing in the cloud are needed. Furthermore, context-aware functionalities need to be implemented, so that data are collected depending on whenever the context (e.g., position, time) is relevant.

- MCS-enabled gateway: since not all the end devices can be MCS-enabled, the MCS-enabled gateway needs to provide the MCS functionalities to common nodes that do not have them. Hence, this gateway provides interfaces so that common nodes can communicate with the MCS components in the cloud, and augment data coming from these nodes with context-aware information.

- Semantic information manager: it is responsible for the management of the semantic data model thanks to which a common semantic description language is used to provide context-awareness to network nodes.

- Big data analysis manager: it receives the data collected by MCS-enabled nodes and gateways and analyses them in order to provide relevant information for the application that needs to be performed by the system.

- Security manager: provides security and privacy functionalities so that system nodes can communicate without any threat, and personal information is kept safe from malicious attacks. 
Table 1. Industrial applications using MCS (mobile crowdsensing and mobile crowdsourcing) and traditional ISNs (Industrial Sensor Networks).

\begin{tabular}{|c|c|c|c|c|c|}
\hline & Application & Acquisition Type & Data Amount & MCS & $\begin{array}{l}\text { Traditional ISN } \\
\end{array}$ \\
\hline \multirow{4}{*}{ Asset utilization } & Asset tracking & Mobile sensors on assets & Medium & Easy thanks to mobility & Possible \\
\hline & Environmental monitoring & $\begin{array}{l}\text { Static and mobile sensors on walls } \\
\text { and assets }\end{array}$ & Medium & Easy thanks to mobility & Easy using static sensors \\
\hline & Energy consumption & Static sensors on assets & Low & Not possible & Easy thanks to static sensors \\
\hline & Fault detection and predictive maintenance & $\begin{array}{l}\text { Static sensors on assets; mobile } \\
\text { sensors on workers' equipment }\end{array}$ & Medium & Easy thanks to mobility & Easy using static sensors \\
\hline \multirow[b]{2}{*}{ Quality control } & Real-time optimization & $\begin{array}{l}\text { Static and mobile sensors on assets } \\
\text { and workers' equipment }\end{array}$ & Medium to large & $\begin{array}{l}\text { Easy thanks to mobility } \\
\text { and scalability }\end{array}$ & Possible thanks to static sensors \\
\hline & Advanced analytics & $\begin{array}{l}\text { Static and mobile sensors on assets, } \\
\text { workers' equipment and workers' } \\
\text { personal devices }\end{array}$ & Large & $\begin{array}{l}\text { Easy thanks to mobility, } \\
\text { scalability and use of } \\
\text { personal devices }\end{array}$ & Limited to static sensors \\
\hline \multirow{4}{*}{ Supply chain management } & Real-time monitoring & $\begin{array}{l}\text { Mobile sensors on products and } \\
\text { vehicles; workers' personal devices }\end{array}$ & Medium to large & $\begin{array}{l}\text { Easy thanks to mobility and } \\
\text { use of personal devices }\end{array}$ & Limited to workers in charge of this task \\
\hline & Logistic tracking & $\begin{array}{l}\text { Mobile sensors on products and } \\
\text { vehicles; workers' personal devices }\end{array}$ & Medium to large & $\begin{array}{l}\text { Easy thanks to mobility and } \\
\text { use of personal devices }\end{array}$ & Limited to workers in charge of this task \\
\hline & Route planning & $\begin{array}{l}\text { Static sensors for roads' condition; } \\
\text { mobile sensors and personal devices } \\
\text { on vehicles }\end{array}$ & Medium to large & $\begin{array}{l}\text { Easy thanks to mobility and } \\
\text { use of personal devices }\end{array}$ & Limited to static sensors \\
\hline & Quality checking & $\begin{array}{l}\text { Mobile sensors on products and } \\
\text { vehicles }\end{array}$ & Medium to large & Easy thanks to mobility & Limited to workers in charge of this task \\
\hline \multirow{6}{*}{ Product monitoring } & Real-time monitoring & $\begin{array}{l}\text { Mobile sensors on products and } \\
\text { vehicles; workers' personal devices }\end{array}$ & Medium to large & $\begin{array}{l}\text { Easy thanks to mobility and } \\
\text { use of personal devices }\end{array}$ & Limited to workers in charge of this task \\
\hline & Logistic tracking & $\begin{array}{l}\text { Mobile sensors on products and } \\
\text { vehicles; workers' personal devices }\end{array}$ & Medium to large & $\begin{array}{l}\text { Easy thanks to mobility and } \\
\text { use of personal devices }\end{array}$ & Limited to workers in charge of this task \\
\hline & Quality checking & Mobile sensors on products & Medium to large & Easy thanks to mobility & Limited to workers in charge of this task \\
\hline & Customer feedback & Customers' mobile personal devices & Medium to large & $\begin{array}{l}\text { Easy thanks to mobility and } \\
\text { use of personal devices }\end{array}$ & Difficult and expensive \\
\hline & Market analysis & Customers' mobile personal devices & Large & $\begin{array}{l}\text { Easy thanks to mobility and } \\
\text { use of personal devices }\end{array}$ & Difficult, expensive and slow \\
\hline & Data-driven demand prediction & Customers' mobile personal devices & Large & $\begin{array}{l}\text { Easy thanks to mobility and } \\
\text { use of personal devices }\end{array}$ & Difficult and slow \\
\hline \multirow{2}{*}{ Workplace safety } & Personal monitoring & $\begin{array}{l}\text { Mobile workers' personal devices } \\
\text { and equipment }\end{array}$ & Medium & Easy thanks to mobility & Difficult \\
\hline & Environmental monitoring & $\begin{array}{l}\text { Static and mobile sensors on walls } \\
\text { and assets }\end{array}$ & Medium & Easy thanks to mobility & Easy using static sensors \\
\hline
\end{tabular}




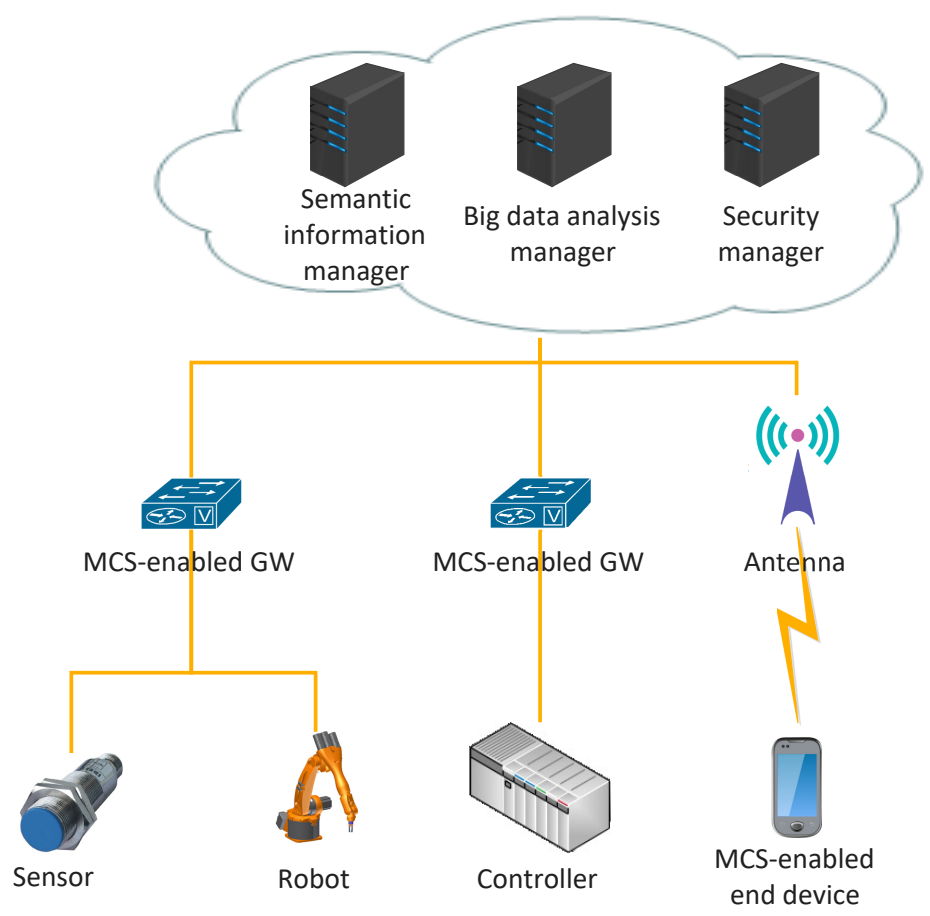

Figure 4. Example MCS-enabled architecture for Industry 4.0.

\subsection{Challenges and Open Issues}

Although Industry 4.0 could greatly benefit from the use of MCS, as proved by the number of industrial applications that would be easily enabled according to Table 1, there are still some open issues that need to be addressed.

The use of personal data coming from personal devices introduces security and privacy issues, particularly with reference to workers' and customers' location. It is extremely important that personal data are secured and leakages are prevented. Nevertheless, this issue tends to fade once data are aggregated.

Although traditional ISNs are usually more expensive and less scalable, they are also more robust and reliable: in ISNs, once sensors are installed, the covered area is guaranteed to be monitored, provided that there are no failures. With MCS, the covered area cannot be known a priori nor guaranteed. Furthermore, the quality of measurement is usually lower.

Handling big amounts of data that need to be transmitted, processed and stored, resource allocation becomes a crucial aspect to be considered, especially considering that battery-powered devices are used. Task assignment techniques need to be implemented [55], so that mobile devices, particularly personal devices, are not overloaded.

Finally, considering only mobile crowdsourcing, recruiting and retaining mechanisms for workers and customers that can provide relevant feedback need to be studied, along with techniques to incentivize them when they complete a task [56]. The drawback that cannot be overlooked is that, when incentives are given, people tend to complete as many tasks as possible, even giving inaccurate information or reducing workers' efficiency.

\section{Conclusions}

In this paper, a comprehensive study of the main technologies that are expected to enable the fourth industrial revolution is presented. The main elements that will foster the spread of Industry 4.0 applications, i.e., ubiquitous Internet access, M2M communication and advanced analytics, are first investigated. Then, the main applications that are expected to be enabled by Industry 4.0 are surveyed. Five application domains have been identified: asset utilization, quality control in manufacturing, 
supply chain management, product monitoring, and workplace safety. Based on the previous analysis, the challenges that are expected before Industry 4.0 can actually see the light, and the opportunities that will be made available are analysed.

From the previous considerations, it has become evident that the impressive amount of data coming from the crowd of sensors and personal devices that characterise the industrial environment can be used to achieve in-depth understanding, gain insights and perform discoveries for accurate decision-making. Therefore, the use of crowdsensing and crowdsourcing represents a benefit for Industry 4.0. The introduced advantages are significant: mobile and scalable measures are provided; new areas can be monitored without the need for additional dedicated devices to be installed; subjective assessments can be easily and cost-effectively collected; human wisdom can be straightforwardly integrated into machine intelligence; information and decision-making processes can be shared among the whole industrial community. Nevertheless, as discussed in the final part of the paper, there are still some open issues that need to be addressed before MCS can be effectively integrated into Industry 4.0.

Acknowledgments: This work was supported in part by the Italian Ministry of University and Research (MIUR), within the Smart Cities framework (Project: CagliariPort2020, ID: SCN_00281).

Conflicts of Interest: The author declares no conflict of interest.

\section{References}

1. Atzori, L.; Iera, A.; Morabito, G. The internet of things: A survey. Comput. Netw. 2010, 54, 2787-2805.

2. Stankovic, J.A. Research directions for the internet of things. IEEE Internet Things J. 2014, 1, 3-9.

3. Evans, P.C.; Annunziata, M. Industrial Internet: Pushing the Boundaries of Minds and Machines. General Electric Reports. 2012. Available online: http:/ / futureview.itrm.ru/documents/50bcf5f13ed696cd87000001. pdf (access on 27 February 2018).

4. Da Xu, L.; He, W.; Li, S. Internet of things in industries: A survey. IEEE Trans. Ind. Inform. 2014, 10, $2233-2243$.

5. Maglaras, L.; Shu, L.; Maglaras, A.; Jiang, J.; Janicke, H.; Katsaros, D.; Cruz, T.J. Industrial Internet of Things (I2oT). Mob. Netw. Appl. 2017, 1-3, doi:10.1007/s11036-017-0937-3.

6. Lee, J.; Bagheri, B.; Kao, H.A. A cyber-physical systems architecture for industry 4.0-based manufacturing systems. Manuf. Lett. 2015, 3, 18-23.

7. Zhou, K.; Liu, T.; Zhou, L. Industry 4.0: Towards future industrial opportunities and challenges. In Proceedings of the IEEE 2015 12th International Conference on Fuzzy Systems and Knowledge Discovery (FSKD), Zhangjiajie, China, 15-17 August 2015; pp. 2147-2152.

8. Jazdi, N. Cyber physical systems in the context of Industry 4.0. In Proceedings of the 2014 IEEE International Conference on Automation, Quality and Testing, Robotics, Cluj-Napoca, Romania, 22-24 May 2014; pp. 1-4.

9. Brettel, M.; Friederichsen, N.; Keller, M.; Rosenberg, M. How virtualization, decentralization and network building change the manufacturing landscape: An industry 4.0 perspective. Int. J. Mech. Ind. Sci. Eng. 2014, 8, 37-44.

10. Shrouf, F.; Ordieres, J.; Miragliotta, G. Smart factories in Industry 4.0: A review of the concept and of energy management approached in production based on the Internet of Things paradigm. In Proceedings of the 2014 IEEE International Conference on Industrial Engineering and Engineering Management (IEEM), Bandar Sunway, Malaysia, 9-12 December 2014; pp. 697-701.

11. Wang, Z.; Chen, C.; Guo, B.; Yu, Z.; Zhou, X. Internet plus in China. IT Prof. 2016, 18, 5-8.

12. Shu, L.; Chen, Y.; Huo, Z.; Bergmann, N.; Wang, L. When mobile crowd sensing meets traditional industry. IEEE Access 2017, 5, 15300-15307.

13. Kagermann, H.; Helbig, J.; Hellinger, A.; Wahlster, W. Recommendations for Implementing the Strategic Initiative INDUSTRIE 4.0: Securing the Future of German Manufacturing Industry; Final Report of the Industrie 4.0 Working Group; Acatech: München, Germany, 2013.

14. Da Silva, G.C.; Kaminski, P.C. From Embedded Systems (ES) to Cyber-Physical Systems (CPS): An Analysis of Transitory Stage of Automotive Manufacturing in the Industry 4.0 Scenario; Technical Report, SAE Technical Paper; SAE International: Warrendale, PA, USA, 2016.

15. Weyrich, M.; Schmidt, J.P.; Ebert, C. Machine-to-machine communication. IEEE Softw. 2014, 31, 19-23. 
16. Varghese, A.; Tandur, D. Wireless requirements and challenges in Industry 4.0. In Proceedings of the IEEE 2014 International Conference on Contemporary Computing and Informatics (IC3I), Mysore, India, 27-29 November 2014; pp. 634-638.

17. Li, X.; Li, D.; Wan, J.; Vasilakos, A.V.; Lai, C.F.; Wang, S. A review of industrial wireless networks in the context of industry 4.0. Wirel. Netw. 2017, 23, 23-41.

18. Gorecky, D.; Schmitt, M.; Loskyll, M.; Zühlke, D. Human-machine-interaction in the Industry 4.0 era. In Proceedings of the 2014 12th IEEE International Conference on Industrial Informatics (INDIN), Porto Alegre, Brazil, 27-30 July 2014; pp. 289-294.

19. Seo, D.; Jeon, Y.B.; Lee, S.H.; Lee, K.H. Cloud computing for ubiquitous computing on M2M and IoT environment mobile application. Clust. Comput. 2016, 19, 1001-1013.

20. Pilloni, V.; Atzori, L.; Mallus, M. Dynamic involvement of real world objects in the IoT: A consensus-based cooperation approach. Sensors 2017, 17, 484, doi:10.3390/s17030484.

21. Bonomi, F.; Milito, R.; Zhu, J.; Addepalli, S. Fog computing and its role in the internet of things. In Proceedings of the First Edition of the MCC Workshop on Mobile Cloud Computing, Helsinki, Finland, 17 August 2012; pp. 13-16.

22. Fogliatto, F.S.; Da Silveira, G.J.; Borenstein, D. The mass customization decade: An updated review of the literature. Int. J. Prod. Econ. 2012, 138, 14-25.

23. Guo, B.; Yu, Z.; Zhou, X.; Zhang, D. From participatory sensing to mobile crowd sensing. In Proceedings of the 2014 IEEE International Conference on Pervasive Computing and Communications Workshops (PERCOM Workshops), Budapest, Hungary, 24-28 March 2014; pp. 593-598.

24. Peng, J.; Zhu, Y.; Shu, W.; Wu, M.Y. When data contributors meet multiple crowdsourcers: Bilateral competition in mobile crowdsourcing. Comput. Netw. 2016, 95, 1-14.

25. Rüßmann, M.; Lorenz, M.; Gerbert, P.; Waldner, M.; Justus, J.; Engel, P.; Harnisch, M. Industry 4.0: The Future of Productivity and Growth in Manufacturing Industries; Boston Consulting Group: Boston, MA, USA, 2015; Volume 9.

26. Karre, H.; Hammer, M.; Kleindienst, M.; Ramsauer, C. Transition towards an Industry 4.0 state of the LeanLab at Graz University of Technology. Procedia Manuf. 2017, 9, 206-213.

27. Robert, J.; Lindner, T.; Milosiu, H. Sub $10 \mu \mathrm{W}$ wake-up-receiver based indoor/outdoor asset tracking system. In Proceedings of the 2015 IEEE 20th Conference on Emerging Technologies \& Factory Automation (ETFA), Luxembourg, 8-11 September 2015; pp. 1-3.

28. Reid, M.; Cook, B. The Application of Smart, Connected Power Plant Assets for Enhanced Condition Monitoring and Improving Equipment Reliability. In Proceedings of the ASME 2016 Power Conference Collocated with the ASME 2016 10th International Conference on Energy Sustainability and the ASME 2016 14th International Conference on Fuel Cell Science, Engineering and Technology, Charlotte, NC, USA, 26-30 June 2016; American Society of Mechanical Engineers (ASME): New York, NY, USA, 2016; p. V001T05A006.

29. Reis, M.S.; Gins, G. Industrial Process Monitoring in the Big Data/Industry 4.0 Era: from Detection, to Diagnosis, to Prognosis. Processes 2017, 5, 35, doi:10.3390/pr5030035.

30. Smajic, H.; Wessel, N. Remote Control of Large Manufacturing Plants Using Core Elements of Industry 4.0. In Online Engineering \& Internet of Things; Springer: Cham, Switzerland, 2018; pp. 546-551.

31. Qian, F.; Zhong, W.; Du, W. Fundamental Theories and Key Technologies for Smart and Optimal Manufacturing in the Process Industry. Engineering 2017, 3, 154-160.

32. Evans, J.R.; Lindsay, W.M. An Introduction to Six Sigma and Process Improvement; Cengage Learning: Boston, MA, USA, 2014.

33. Lee, J.; Kao, H.A.; Yang, S. Service innovation and smart analytics for industry 4.0 and big data environment. Procedia Cirp 2014, 16, 3-8.

34. Li, J.; Tao, F.; Cheng, Y.; Zhao, L. Big data in product lifecycle management. Int. J. Adv. Manuf. Technol. 2015, 81, 667-684.

35. Barreto, L.; Amaral, A.; Pereira, T. Industry 4.0 implications in logistics: An overview. Procedia Manuf. 2017, 13, 1245-1252.

36. Mallus, M.; Colistra, G.; Atzori, L.; Murroni, M.; Pilloni, V. Dynamic Carpooling in Urban Areas: Design and Experimentation with a Multi-Objective Route Matching Algorith. Sustainability 2017, 9, 254, doi:10.3390/su9020254. 
37. Zhong, R.Y.; Xu, X.; Wang, L. IoT-enabled Smart Factory Visibility and Traceability using Laser-scanners. Procedia Manuf. 2017, 10,1-14.

38. Scheuermann, C.; Verclas, S.; Bruegge, B. Agile factory-An example of an industry 4.0 manufacturing process. In Proceedings of the 2015 IEEE 3rd International Conference on Cyber-Physical Systems, Networks, and Applications (CPSNA), Hong Kong, China, 19-21 August 2015; pp. 43-47.

39. Yan, J.; Meng, Y.; Lu, L.; Li, L. Industrial Big Data in an Industry 4.0 Environment: Challenges, Schemes, and Applications for Predictive Maintenance. IEEE Access 2017, 5, 23484-23491.

40. Roßmann, B.; Canzaniello, A.; von der Gracht, H.; Hartmann, E. The future and social impact of Big Data Analytics in Supply Chain Management: Results from a Delphi study. Technol. Forecast. Soc. Chang. 2017, doi:10.1016/j.techfore.2017.10.005.

41. Xu, X.; Zhong, M.; Wan, J.; Yi, M.; Gao, T. Health monitoring and management for manufacturing workers in adverse working conditions. J. Med. Syst. 2016, 40, 222, doi:10.1007/s10916-016-0584-4.

42. Behr, C.; Kumar, A.; Hancke, G. A smart helmet for air quality and hazardous event detection for the mining industry. In Proceedings of the 2016 IEEE International Conference on Industrial Technology (ICIT), Taipei, Taiwan, 14-17 March 2016; pp. 2026-2031.

43. Kulkarni, P.; Sangam, V. Smart Helmet for Hazardous event Detection and Evaluation in mining Industries using wireless communication. J. Commun. Eng. Innov. 2017, 3, 11-16.

44. Wollschlaeger, M.; Sauter, T.; Jasperneite, J. The future of industrial communication: Automation networks in the era of the internet of things and industry 4.0. IEEE Ind. Electron. Mag. 2017, 11, 17-27.

45. Nitti, M.; Pilloni, V.; Colistra, G.; Atzori, L. The Virtual Object as a Major Element of the Internet of Things: A Survey. IEEE Commun. Surv. Tutor. 2015, 18, 1228-1240.

46. Shin, M.; Cornelius, C.; Kapadia, A.; Triandopoulos, N.; Kotz, D. Location privacy for mobile crowd sensing through population mapping. Sensors 2015, 15, 15285-15310.

47. Schmidt, R.; Möhring, M.; Härting, R.C.; Reichstein, C.; Neumaier, P.; Jozinović, P. Industry 4.0-potentials for creating smart products: Empirical research results. In Proceedings of the International Conference on Business Information Systems, Poznań, Poland, 24-26 June 2015; Springer: Cham, Switzerland, 2015; pp. 16-27.

48. Basanta-Val, P. An efficient industrial big-data engine. IEEE Trans. Ind. Inform. 2017, doi:10.1109/TII.2017.2755398.

49. Lv, Z.; Song, H.; Basanta-Val, P.; Steed, A.; Jo, M. Next-generation big data analytics: State of the art, challenges, and future research topics. IEEE Trans. Ind. Inform. 2017, 13, 1891-1899.

50. Congosto, M.; Basanta-Val, P.; Sanchez-Fernandez, L. T-Hoarder: A framework to process Twitter data streams. J. Netw. Comput. Appl. 2017, 83, 28-39.

51. Shu, L.; Mukherjee, M.; Pecht, M.; Crespi, N.; Han, S.N. Challenges and Research Issues of Data Management in IoT for Large-Scale Petrochemical Plants. IEEE Syst. J. 2017, doi:10.1109/JSYST.2017.2700268.

52. Conti, M.; Passarella, A.; Das, S.K. The Internet of People (IoP): A new wave in pervasive mobile computing. Pervasive Mob. Comput. 2017, 41, 1-27.

53. Baccarelli, E.; Naranjo, P.G.V.; Scarpiniti, M.; Shojafar, M.; Abawajy, J.H. Fog of everything: Energy-efficient networked computing architectures, research challenges, and a case study. IEEE Access 2017, 5, 9882-9910.

54. Hu, X.; Li, X.; Ngai, E.; Leung, V.; Kruchten, P. Multidimensional context-aware social network architecture for mobile crowdsensing. IEEE Commun. Mag. 2014, 52, 78-87.

55. Colistra, G.; Pilloni, V.; Atzori, L. Task allocation in group of nodes in the IoT: A consensus approach. In Proceedings of the 2014 IEEE International Conference on Communications (ICC), Sydney, NSW, Australia, 10-14 June 2014; pp. 3848-3853.

56. Karati, A.; Islam, S.H.; Biswas, G.; Bhuiyan, M.Z.A.; Vijayakumar, P.; Karuppiah, M. Provably Secure Identity-based Signcryption Scheme for Crowdsourced Industrial Internet of Things Environments. IEEE Internet Things J. 2017, doi:10.1109/JIOT.2017.2741580.

(C) 2018 by the author. Licensee MDPI, Basel, Switzerland. This article is an open access article distributed under the terms and conditions of the Creative Commons Attribution (CC BY) license (http:/ / creativecommons.org/licenses/by/4.0/). 